\title{
Photochromic antifolate for light-activated chemotherapy
}

\author{
Carlo Matera ${ }^{\mathrm{a}, \mathrm{b}}$, Alexandre M.J. Gomila ${ }^{\mathrm{a}, \mathrm{b}}$, Núria Camarera ${ }^{\mathrm{a}, \mathrm{b}}$, Michela Libergolia ${ }^{\mathrm{a}}$, Concepció Soler ${ }^{\mathrm{c}}$, \\ and Pau Gorostiza, $\mathrm{a}^{\mathrm{a}, \mathrm{b}, \mathrm{,}, *}$ \\ ${ }^{a}$ Institute for Bioengineering of Catalonia (IBEC), Barcelona Institute for Science and Technology \\ (BIST), Barcelona 08028, Spain; ${ }^{b}$ Biomedical Research Networking Center in Bioengineering, \\ Biomaterials and Nanomedicine (CIBER-BBN), Madrid 28029, Spain; ${ }^{\circ}$ Department of Pathology \\ and Experimental Therapeutics, School of Medicine and Health Sciences, Universitat de Barcelona, \\ IDIBELL, L'Hospitalet de Llobregat 08907, Spain; ${ }^{\mathrm{d}}$ Catalan Institution for Research and Advanced \\ Studies (ICREA), Barcelona 08010, Spain; ); *email: pau@icrea.cat
}

\begin{abstract}
Although cytotoxic chemotherapy is one of the primary pharmacological treatments for chronic hyperproliferative diseases such as cancer and psoriasis, its efficacy and tolerability are in many cases dramatically limited by off-target toxicity. A promising approach to improve these therapies is to activate the drugs exclusively at their desired place of action. In fact, in those diseases that would benefit from a highly localized treatment, a precise spatiotemporal control over the activity of a chemotherapeutic agent would allow reducing the concentration of active compound outside the targeted region, improving the tolerability of the treatment. Light is a powerful tool in this respect: it offers unparalleled opportunities as a non-invasive regulatory signal for pharmacological applications because it can be delivered with high precision regarding space, time, intensity and wavelength. Photopharmacology represents a new and emerging approach in this regard since the energy of light is used to change the structure of the drug and hence to switch its pharmacological activity on and off on demand. We describe here phototrexate, the first light-regulated inhibitor of the human DHFR. Enzyme and cell viability assays demonstrated that phototrexate behaves as a potent antifolate in its cis configuration, obtained under UVA illumination, and that it is nearly inactive in its dark-relaxed trans form. Experiments in zebrafish confirmed that phototrexate can disrupt folate metabolism in a light-dependent fashion also in vivo. Overall, phototrexate represents a potential candidate towards the development of an innovative photoactivated antifolate chemotherapy.
\end{abstract}

Keywords: cancer, dermatology, methotrexate, photoactivated chemotherapy, photodynamic therapy, phototherapy, psoriasis, rheumatoid arthritis

\section{INTRODUCTION}

Chemotherapy is one of the main treatment options for cancer and psoriasis, however chemotherapeutic agents usually suffer from limitations that restrict their use and have a major impact on the patients' quality of life and survival. In particular, the systemic distribution of cytotoxic drugs reduces their efficacy and causes severe side effects due to nonselective toxicity. Therefore, the lack of organ/tissue selectivity remains a major problem. Ineffective treatments cause huge emotional and societal burdens to the patients and their environment, and have a considerable economic impact. ${ }^{1,2} \mathrm{~A}$ promising approach to improve these therapies is to activate the cytotoxic agents exclusively at their desired place of action. In fact, in those diseases that would benefit from a highly localized treatment, a precise spatiotemporal control over the activity of a cytotoxic agent would allow reducing off-target effects, improving the tolerability of the treatment.

Light is a powerful tool in this respect, as it offers unparalleled opportunities as a non-invasive regulatory signal for medical applications because it can be delivered with high precision regarding space, time, intensity and wavelength. ${ }^{3-6}$ The most successful representative of these photo-induced cytotoxic treatments is photodynamic therapy (PDT), which has been shown to be effective in a number of oncologic and non-oncologic conditions with minimal invasivity. ${ }^{7}$ PDT consists in 
administration of a photosensitizer, which undergoes excitation after suitable irradiation emitted from a source of light and generates singlet oxygen and other cytotoxic oxygen species such as superoxide anion radical and hydroxyl radical. However, also PDT has some limitations. Tissue oxygenation is crucial for the photodynamic effect to take place; therefore, extensive necrotic tissue or dense tumor masses can impair PDT. In addition, PDT treatments are in many cases unsuitable options because of the high variability in clinical response and the appearance of unbearable side effects (e.g., severe pain, burning sensation). Furthermore, therapeutic agents can still diffuse to other parts of the body causing off-target effects and toxicity in healthy tissues. ${ }^{8,9}$ Different ways to circumvent such limitations have been proposed, however, there have been limited applications in vivo so far. ${ }^{10,11}$

Photopharmacology ${ }^{12-16}$ represents a new and emerging approach in this regard since the energy of light is used to change the structure of a drug and hence to switch its pharmacological activity on and off on demand. ${ }^{17-19}$ Light-regulated drug candidates can be obtained by modifying clinically approved drugs by the incorporation of photoresponsive molecular switches, thus enabling the reversible modulation of their biological properties upon illumination at specific wavelengths. Herein, we report on the development and characterization of phototrexate, the first photoswitchable inhibitor of the human dihydrofolate reductase and a promising candidate for an innovative photoactivated chemotherapy (Figure $1 \mathrm{~A}) .{ }^{15}$

\section{MATERIALS \& METHODS}

3D alignments and similarity values. 3D overlays between the crystal structure of methotrexate (PDB 1U72) and the structure of phototrexate (trans and cis isomers) were performed with MarvinSketch 17.28.0 by ChemAxon using the "3D Alignment" tool (alignment type: by common scaffold; initial conformation count: 5 ; accuracy: accurate). ${ }^{20}$

Characterization of photochromism. UV/Vis absorption spectra were measured at room temperature with a Shimadzu UV-2501PC UV/Vis spectrophotometer in $10 \mathrm{~mm}$ quartz cuvettes. NMR spectra were acquired on a Varian Mercury $400 \mathrm{MHz}$ spectrometer. HPLC analyses were performed with a Waters Alliance 2795 separation module (RP column: XSelect CSH C18, 50x4.6 mm, S-3.5 $\mu \mathrm{m}, 1.6 \mathrm{ml} / \mathrm{min}$; eluent: from 5\% B to 100\% B in $3.5 \mathrm{~min}$ using a linear gradient, A: $\mathrm{H}_{2} \mathrm{O} 0.1 \%$ formic acid, $\mathrm{B}$ : acetonitrile $0.1 \%$ formic acid) coupled to a Waters 2996 photodiode detector and a Waters 3100 mass spectrometer. Irradiations were performed with LED arrays emitting at $375 \mathrm{~nm}\left(2.6 \mathrm{~mW} / \mathrm{cm}^{2}\right), 395 \mathrm{~nm}\left(3.5 \mathrm{~mW} / \mathrm{cm}^{2}\right)$, and $500 \mathrm{~nm}\left(1.3 \mathrm{~mW} / \mathrm{cm}^{2}\right)$.

Enzyme inhibition assays. DHFR activity was measured using a DHFR assay kit (CS0340, Sigma-Aldrich) as per the manufacturer's instructions with Nunc 96-Well MicroWell microplates (ThermoScientific) and a BMG FLUOstar OPTIMA microplate reader, measuring the decrease in absorbance at $340 \mathrm{~nm}$ (NADPH absorbance) every $15 \mathrm{~s}$ over 2.75 min. Cis-enriched phototrexate was obtained by pre-illuminating a stock solution with $375 \mathrm{~nm}$ light right before each experiment. All experiments were performed in dim light to prevent unwanted photoisomerization. Residual enzyme activity was calculated by comparing the slope of obtained kinetics before and after inhibition. Data are means of at least three independent experiments performed in triplicate \pm SEM.

Cell viability assays. The effect of phototrexate on cell viability was evaluated by an MTT assay using Nunc 96-Well MicroWell microplates (Thermo Scientific) and a Thermo Scientific Multiskan FC microplate reader. MTT (thiazolyl blue tetrazolium bromide) was purchased from Sigma-Aldrich (M5655). Cis-enriched phototrexate was obtained by preilluminating a stock solution with $375 \mathrm{~nm}$ light right before each experiment. HCT116 human colon cancer cells were cultured in DMEM (31885-023, Thermo Scientific) with 10\% fetal bovine serum and 1\% streptomycin/penicillin. Cells were dispensed in a sterile 96 -well plate at a cell density of 5000 cells/well $(160 \mu \mathrm{L} /$ well $)$ and maintained at $37{ }^{\circ} \mathrm{C}$ and $5 \%$ $\mathrm{CO}_{2}$ for 24 hours before treatments. For background wells, no cells were seeded. Then the medium was aspirated and replaced by $160 \mu \mathrm{L}$ of fresh medium containing different concentrations of the test compounds. Control wells received $160 \mu \mathrm{L}$ of fresh medium containing the vehicle. To account for the thermal relaxation of the cis isomer in the test conditions, after 24 hours of incubation at $37{ }^{\circ} \mathrm{C}$ and $5 \% \mathrm{CO}_{2}$ the medium was carefully replaced in each well (for all treatment groups) with the same volume of fresh medium of identical initial composition. After 48 hours of incubation at $37{ }^{\circ} \mathrm{C}$ and $5 \% \mathrm{CO}_{2}$, the medium was carefully removed and each well was washed with $200 \mu \mathrm{L}$ of phosphate buffered saline (PBS). Then $200 \mu \mathrm{L}$ of MTT $(0.5 \mathrm{mg} / \mathrm{mL})$ in PBS were added in the dark to each well and microplates were incubated at $37^{\circ} \mathrm{C}$ for 4 hours. The medium was cautiously aspirated and formazan crystals were dissolved in DMSO (200 $\mu \mathrm{L} /$ well). Microplates were covered with aluminium foil and gently shaken for 15 minutes (orbital shaking), then absorbance was 
measured at $570 \mathrm{~nm}$. Cytotoxicity was expressed as a relative viability of cells compared to control wells. All experiments were performed in dim light to prevent unwanted photoisomerization. Data are means of at least three independent experiments performed in triplicate \pm SEM. Results were analyzed by two-way ANOVA with Bonferroni's multiple comparisons test for statistical significance (GraphPad Prism 6).

Zebrafish assays. Zebrafish (Danio rerio) embryos were used as a model system to evaluate the effects of trans and cis phototrexate in vivo (disruption of animal development). All studies were conducted on wild-type zebrafish larvae (Tupfel long-fin strain) purchased from the animal facility of the Barcelona Biomedical Research Park (PRBB). Experiments on animals were conducted according to the European Directive 2010/63/EU. Zebrafish fertilized eggs within 5 hours post fertilization (hpf) were placed in a 12-well plate (TPP tissue culture test plate, cat. 92024) with $1 \mathrm{~mL}$ of treatment solution per well at $28{ }^{\circ} \mathrm{C}$. Four treatment groups were studied: 1) vehicle (2\% DMSO); 2) MTX $\left.200 \mu \mathrm{M} ; 3\right)$ cis-phototrexate 200 $\mu \mathrm{M}$; 4) trans-phototrexate $200 \mu \mathrm{M}$. Cis-enriched phototrexate was obtained by pre-illuminating a stock solution with 375 nm light right before each experiment. The reference inhibitor MTX (A6770, Sigma-Aldrich) was tested for comparison. All treatment solutions were freshly prepared and replaced every 24 hours during five consecutive days to account for the thermal relaxation of the cis isomer in the test conditions. Each individual was observed daily to monitor anatomical changes. Once larvae were developed, anatomical development was fully analyzed and described. For our purposes, three specific aberrant anatomical traits were observed: one affecting the pigmentation pattern of the larvae, another referring to the volume of the heart cavity, and a third trait which includes the bending or the turning of the larva tail.

\section{RESULTS \& DISCUSSION}

Through an extensive review of scientific literature and databases, we identified the dihydrofolate reductase (DHFR) inhibitor methotrexate (MTX) as one of the most suitable drugs matching the identikit to develop a photoswitchable chemotherapeutic agent (Figure 1B). MTX belongs to a class of drugs known as antimetabolites and is used to treat certain types of cancer or to control other diseases characterized by abnormal cell proliferation, such as severe psoriasis or rheumatoid arthritis that have not responded to other treatments. ${ }^{21}$ Furthermore, its chemical structure is amenable to "azologization", which is a strategy to convert an established drug into a photoswitchable one through an isosteric replacement and hence with minimal structural perturbation of the pharmacophore. ${ }^{13,22}$ Another reason for selecting MTX as our parent drug was that, in the crystal structure of its ternary complex with the human DHFR and NADPH, the conformation of MTX is bent at the N-methylaminomethylene group that links the two aromatic moieties in the drug structure (Figure 1D, PDB 1U72). Thus, a plain azologue obtained by replacement of the methylaminomethylene group with an azo group is expected to be inactive in the most thermodynamically stable configuration (trans isomer) and active in the less stable configuration, which is normally desirable because ideally such a photoswitchable drug could be safely administered -even systemically- in the inactive relaxed form, and then be photoactivated on demand at the required locations and times. We could then finally design our photochromic MTX analogue, and named it phototrexate (PHX). In order to facilitate the chemical synthesis of the envisaged photoswitchable antifolate, we also conveniently planned to replace the pteridine nucleus of MTX with a quinazoline one, since it is reported that this substitution should not significantly affect the affinity of the ligand for the target enzyme (Figure 1C). ${ }^{23}$ As expected, superimposition of phototrexate and MTX showed that only the cis isomer of phototrexate should mimic the bent conformation of MTX bound to the hDHFR (with a 3D similarity score of 0.64 versus 0.28 , calculated with MarvinSketch; Figure $1 \mathrm{E}$ ). 


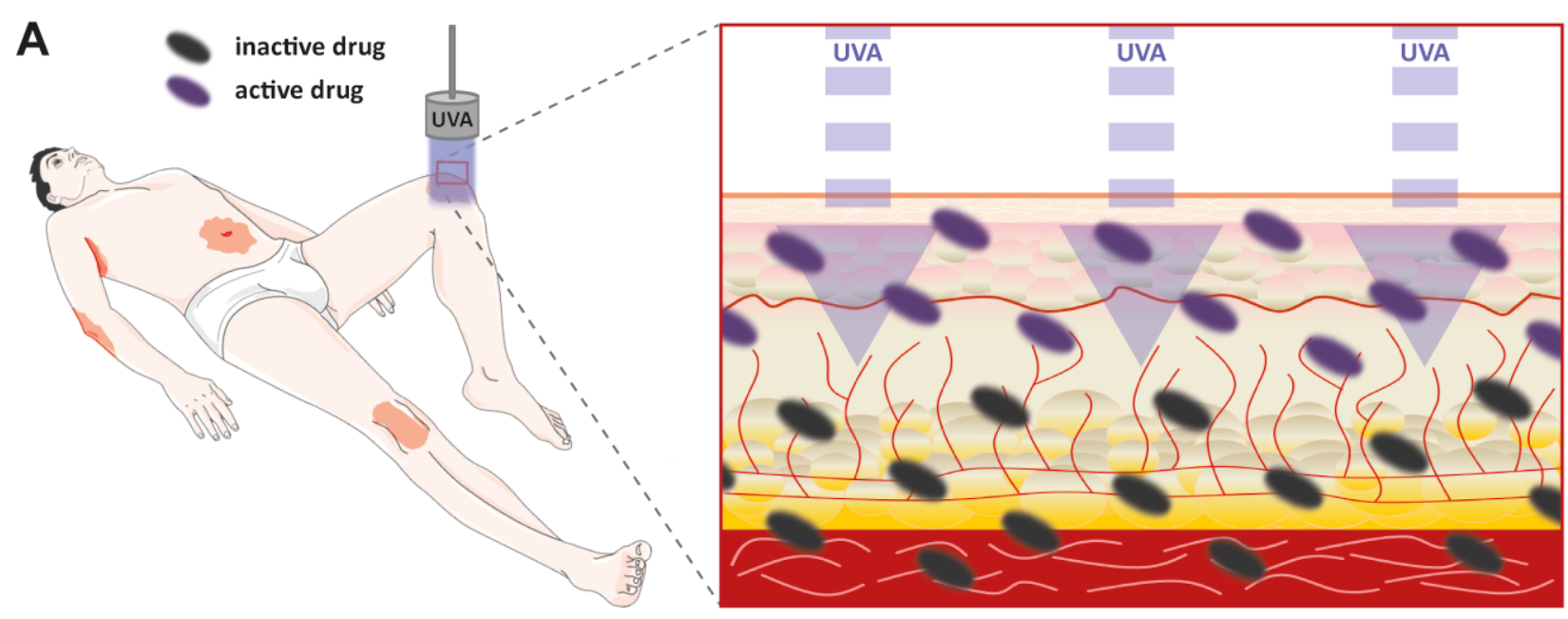

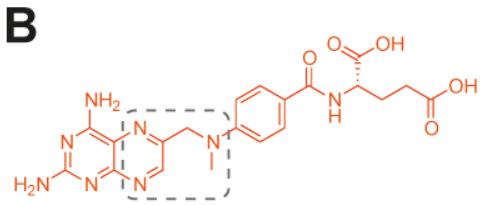

methotrexate

D

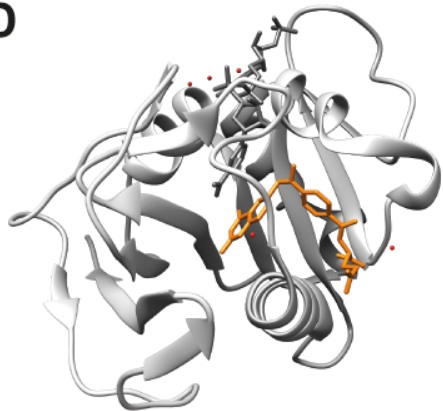

C<smiles>CCCCCCCC(=O)O</smiles>

trans-phototrexate

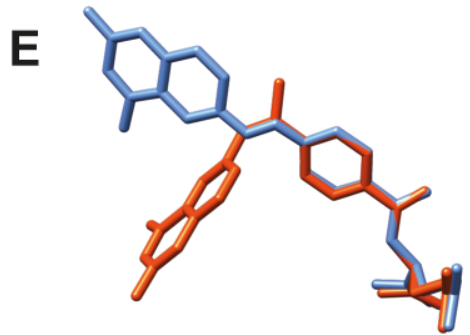

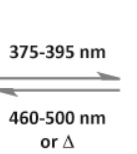

or $\Delta$

(1)

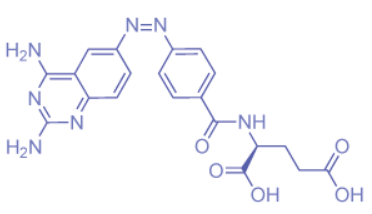

cis-phototrexate

Figure 1. The envisaged photoactivated chemotherapy and design strategy of phototrexate. (A) A photoswitchable chemotherapeutic agent could be administered in the inactive form and activated with light only where and when needed. Since the photoswitching is reversible, the drug would spontaneously self-deactivate upon diffusing away from the illuminated site by regaining its "constitutive" inactive conformation, thus reducing the occurrence of unwanted effects in off-target regions. (B) Chemical structure of the DHFR inhibitor methotrexate. The dotted rectangle defines the part of the molecule involved in the azologization strategy. (C) Chemical structures of phototrexate in its trans and cis configurations. (D) Crystal structure of the human DHFR (gray) in complex with methotrexate (orange) and the cofactor NADPH (dark gray) (PDB 1U72). (E) Superimposition of methotrexate (crystal structure, orange), trans-phototrexate (blue) and cis-phototrexate (purple). Unlike trans-phototrexate, cis-phototrexate mimics the bent conformation of methotrexate bound to the DHFR.

Phototrexate was prepared as previously reported via a convergent 4-step synthesis starting from commercially available building blocks. ${ }^{15,24}$ An essential requirement for using phototrexate as a photoswitchable antifolate is that it efficiently responds to light, which means that it can be promptly photoisomerized between its two different configurations with a relatively high degree of photoconversion (cis/trans ratio). UV/Vis absorption studies showed that phototrexate can be effectively isomerized from trans to cis with UVA light (375-395 nm) and back-isomerized from cis to trans with visible 
light (460-500 nm) (Figure 2A). The extent of photoconversion was quantified by ${ }^{1} \mathrm{H}-\mathrm{NMR}$ analysis. We found out that the composition of the photostationary state changed from $99-100 \%$ trans to $86 \%$ cis after 15 -min irradiation with $395 \mathrm{~nm}$ light (Figure 2B). In addition, we also observed by HPLC analysis that phototrexate can spontaneously relaxes back in the dark from the cis active to the trans inactive form in a few hours at $37{ }^{\circ} \mathrm{C}$ (human body temperature) in aqueous environment (Figure 2C). Overall, we concluded that the photochromic behavior of phototrexate was suitable for our purposes and characterized then its photopharmacological properties.
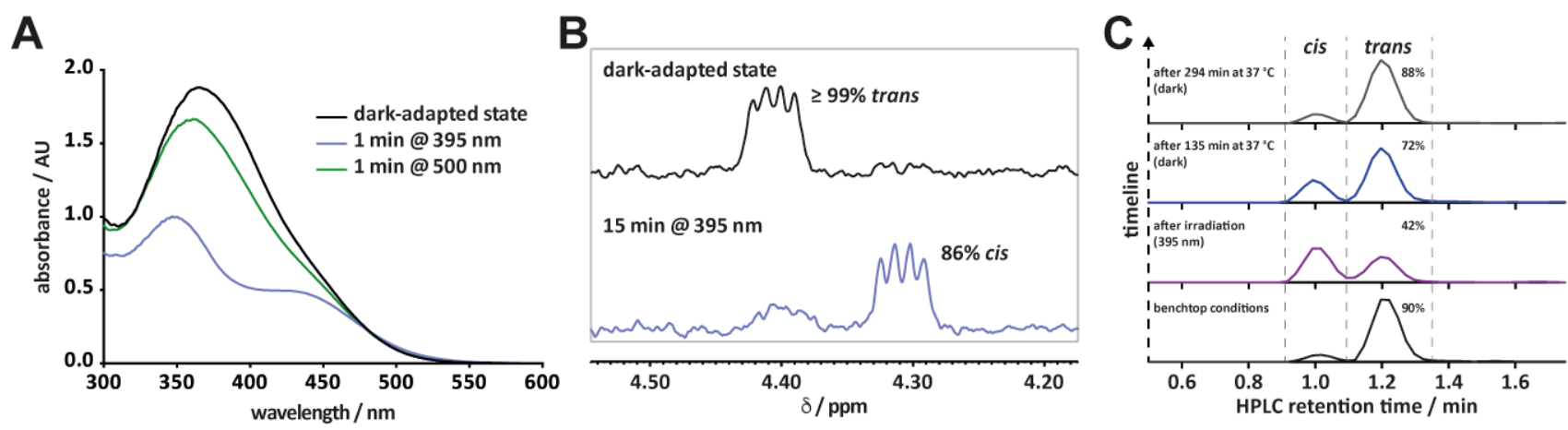

Figure 2. Photochemical characterization of phototrexate. (A) UV/Vis absorption spectra of phototrexate $(100 \mu \mathrm{M}$ in $\mathrm{NaOH}$ $100 \mu \mathrm{M}$ ) showing distinct photochromic behavior. (B) Composition of the photostationary state achieved after illumination with $395 \mathrm{~nm}$ light for $15 \mathrm{~min}$ determined by ${ }^{1} \mathrm{H}-\mathrm{NMR}\left(1 \mathrm{mM}\right.$ in $\left.\mathrm{D}_{2} \mathrm{O} / \mathrm{NaOH}\right)$. (C) Thermal relaxation of phototrexate (from cis to trans, $100 \mu \mathrm{M}$ in $\mathrm{NaOH} 100 \mu \mathrm{M})$ in the dark at $37^{\circ} \mathrm{C}$ observed by HPLC-MS analysis.

First, we assessed with a colorimetric assay that phototrexate isomers can inhibit the DHFR with different efficacy. In the presence of $10 \mathrm{nM}$ trans-phototrexate, only a moderate inhibition of the enzyme activity was observed (78\% residual activity), whereas, in the presence of the cis-enriched phototrexate, the enzyme reaction was almost completely inhibited (22\% residual activity) (Figure 3A). These results demonstrated that cis-phototrexate is a much stronger DHFR inhibitor and are in agreement with our design of a "cis-on" antifolate photoswitchable drug.

We next examined the cytotoxicity of phototrexate in cells using an MTT assay for cell viability. HCT116 human colon cancer cells were incubated with trans-phototrexate and cis-enriched phototrexate for $48 \mathrm{~h}$ at $37^{\circ} \mathrm{C}$, and cell viability was subsequently measured. In line with the results obtained in enzyme inhibition assays, the cis isomer was significantly more toxic to the HCT116 cells than the trans (Figure 3B). At $0.1 \mu \mathrm{M}$, the cis-enriched compound killed $45 \%$ of the cells, whereas the trans killed only $11 \%$ of the cells. At $1 \mu \mathrm{M}$, the cis-enriched form killed $73 \%$ of the cells, versus the $42 \%$ obtained with the pure trans compound. This difference between the two isomers is even more remarkable if we consider the relatively low thermal stability of the cis form in relation to the duration of the whole experiment (48 h). Phototrexate cytotoxicity can be reasonably ascribed to the disruption of the folate pathway since this metabolic process plays a fundamental role in the process of cell division.

Finally, we wanted to observe the effects of our compound in a small animal model. Because of its antifolate activity, phototrexate should remarkably perturb early stages of animal development. For this purpose, we tested phototrexate in zebrafish (Danio rerio), which has been recognized as an excellent model system to investigate the effects of antifolate drugs in vivo. ${ }^{25}$ Zebrafish fertilized eggs were incubated within $5 \mathrm{~h}$ post fertilization (hpf) in water containing transphototrexate or cis-enriched phototrexate $(200 \mu \mathrm{M})$ for 5 days. MTX was tested as the positive control at the same concentration. The four groups were compared at $72 \mathrm{hpf}$, when certain anatomical traits become easier to observe (Figure 3C). In the vehicle group no malformations were observed, except for a non-hatched individual with an unformed tail. In the group treated with MTX, all the survivors presented three abnormalities at $72 \mathrm{hpf:1}$ ) an abnormal volume of the cardiac cavity; 2) tail angle deviations, with prominent bent or curved tails; 3) a deficient iridiophore ocular pigmentation, with 
complete black colored eyes and a deficient blackbody stripe pattern. In the cis-phototrexate group a toxicity profile similar to that of the reference compound MTX was observed, with comparable developmental abnormalities at $72 \mathrm{hpf}$. In stark contrast, trans-phototrexate showed a much lower toxicity, as no abnormalities were observed in this group at $72 \mathrm{hpf}$ (Figure 3C). Overall, our in vivo experiment showed that the photoactivated (cis) form of our compound behaves as a potent antifolate drug, while it is almost inactive in its thermodynamically more stable (trans) configuration.

A

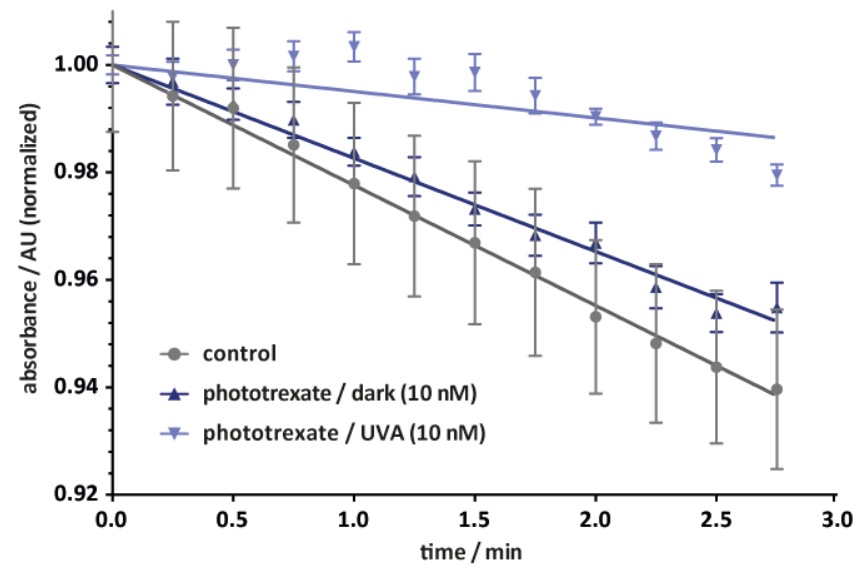

C

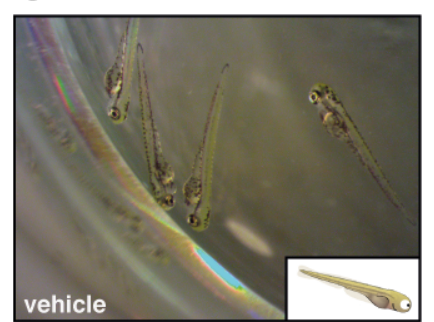

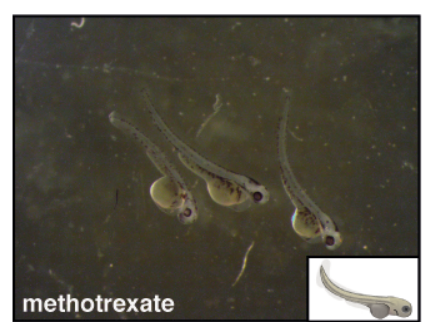

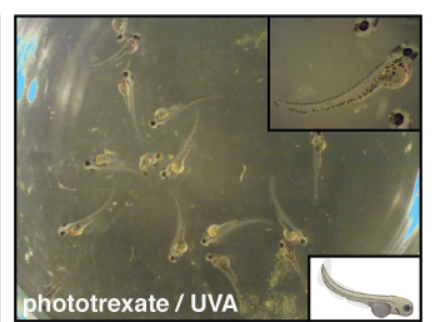

B

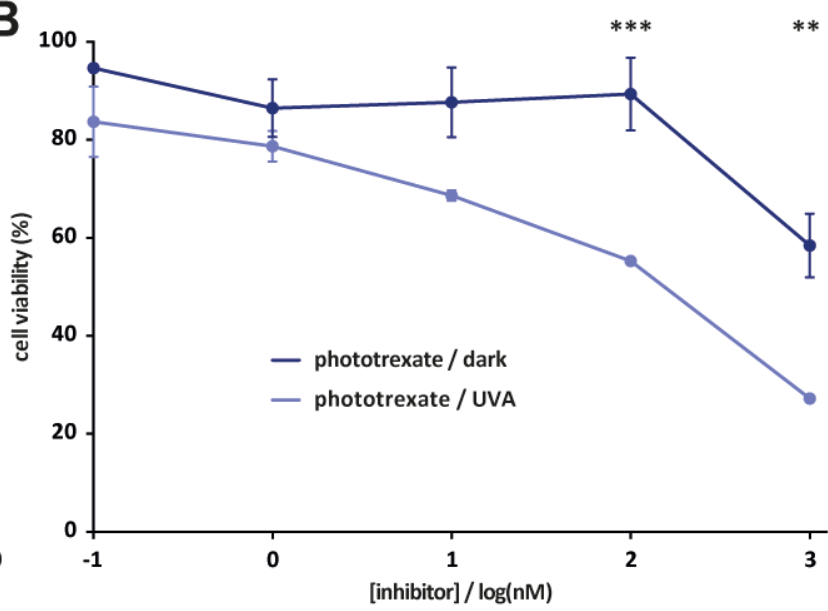

Figure 3. Photopharmacological characterization of phototrexate. (A) Inhibition of DHFR activity at $10 \mathrm{nM}$ of trans (blue) and cis (purple) phototrexate versus the control. (B) HCT116 cell viability assay at different concentrations of trans- and cisphototrexate. (C) Effects of methotrexate and phototrexate $(200 \mu \mathrm{M})$ on zebrafish development. The pictures illustrate the common abnormal developmental traits in the methotrexate and cis-phototrexate (obtained by UVA illumination) groups, in contrast with the vehicle and trans-phototrexate groups.

\section{CONCLUSIONS}

In conclusion, we have developed phototrexate, the first photoswitchable analogue of methotrexate, and demonstrated its antifolate and antiproliferative properties in vitro and in vivo. Our results showed that phototrexate can be switched on with UVA light, and self-deactivates by thermal relaxation in the dark. It can also be deactivated with visible light. Thus, phototrexate constitutes a proof-of-concept to design photoswitchable chemotherapeutic agents and a drug candidate towards the development of a photoactivated antifolate chemotherapy. Molecules like phototrexate could be administered in their inactive form, and then be photoactivated on demand only where and when needed. Suitable target organs and 
tissues might be all those accessible to illumination either directly or through natural orifices (e.g., skin, bladder, intestine). In addition, the envisioned photochemotherapy may also benefit from medical devices already in use for PDT. The safety of phototrexate is currently under assessment, and several potential applications are under consideration, such as psoriasis, different forms of cancer, and other hyperproliferative and inflammatory diseases.

\section{REFERENCES}

[1] Chabner, B. A., Roberts, T. G., "Chemotherapy and the war on cancer," Nat Rev Cancer 5(1), 65-72, Nature Publishing Group (2005).

[2] Miller, K. D., Siegel, R. L., Lin, C. C., Mariotto, A. B., Kramer, J. L., Rowland, J. H., Stein, K. D., Alteri, R., Jemal, A., "Cancer treatment and survivorship statistics, 2016," CA: A Cancer Journal for Clinicians 66(4), 6 ed., 271-289 (2016).

[3] Mayer, G., Heckel, A., Biologically active molecules with a "light switch"., Angew. Chem. Int. Ed. Engl. 45(30), 4900-4921 (2006).

[4] Beharry, A. A., Woolley, G. A., “Azobenzene photoswitches for biomolecules.," Chem Soc Rev 40(8), 4422-4437, The Royal Society of Chemistry (2011).

[5] Brieke, C., Rohrbach, F., Gottschalk, A., Mayer, G., Heckel, A., "Light-controlled tools.,” Angew. Chem. Int. Ed. Engl. 51(34), 8446-8476, WILEY-VCH Verlag (2012).

[6] Szymanski, W., Beierle, J. M., Kistemaker, H. A. V., Velema, W. A., Feringa, B. L., "Reversible photocontrol of biological systems by the incorporation of molecular photoswitches.," Chem. Rev. 113(8), 6114-6178 (2013).

[7] Mallidi, S., Anbil, S., Bulin, A.-L., Obaid, G., Ichikawa, M., Hasan, T., "Beyond the Barriers of Light Penetration: Strategies, Perspectives and Possibilities for Photodynamic Therapy.," Theranostics 6(13), 2458-2487 (2016).

[8] Choi, Y. M., Adelzadeh, L., Wu, J. J., "Photodynamic therapy for psoriasis.," J Dermatolog Treat 26(3), 202-207 (2015).

[9] Hamblin, M. R., Huang, Y.-Y., Handbook of Photomedicine, CRC Press (2013).

[10] Sgambellone, M. A., David, A., Garner, R. N., Dunbar, K. R., Turro, C., "Cellular toxicity induced by the photorelease of a caged bioactive molecule: design of a potential dual-action Ru(II) complex.," J. Am. Chem. Soc. 135(30), 11274-11282 (2013).

[11] Scherer, K. M., Bisby, R. H., Botchway, S. W., Hadfield, J. A., Parker, A. W., “Anticancer phototherapy using activation of E-combretastatins by two-photon-induced isomerization.," J Biomed Opt 20(5), 051004, International Society for Optics and Photonics (2015).

[12] Nevola, L., Martín-Quirós, A., Eckelt, K., Camarero, N., Tosi, S., Llobet, A., Giralt, E., Gorostiza, P., "Lightregulated stapled peptides to inhibit protein-protein interactions involved in clathrin-mediated endocytosis.," Angew. Chem. Int. Ed. Engl. 52(30), 7704-7708, WILEY-VCH Verlag (2013).

[13] Pittolo, S., Gómez-Santacana, X., Eckelt, K., Rovira, X., Dalton, J., Goudet, C., Pin, J.-P., Llobet, A., Giraldo, J., et al., "An allosteric modulator to control endogenous G protein-coupled receptors with light.," Nat. Chem. Biol. 10(10), 813-815, Nature Publishing Group (2014).

[14] Izquierdo-Serra, M., Bautista-Barrufet, A., Trapero, A., Garrido-Charles, A., Díaz-Tahoces, A., Camarero, N., Pittolo, S., Valbuena, S., Pérez-Jiménez, A., et al., "Optical control of endogenous receptors and cellular excitability using targeted covalent photoswitches.," Nature Communications 7(1), 12221, Nature Publishing Group (2016).

[15] Matera, C., Gomila, A. M. J., Camarero, N., Libergoli, M., Soler, C., Gorostiza, P., "Photoswitchable Antimetabolite for Targeted Photoactivated Chemotherapy.," J. Am. Chem. Soc. 140(46), 15764-15773 (2018).

[16] Riefolo, F., Matera, C., Garrido-Charles, A., Gomila, A. M. J., Sortino, R., Agnetta, L., Claro, E., Masgrau, R., Holzgrabe, U., et al., "Optical Control of Cardiac Function with a Photoswitchable Muscarinic Agonist.," J. Am. Chem. Soc. 141(18), 7628-7636 (2019).

[17] Velema, W. A., Szymanski, W., Feringa, B. L., "Photopharmacology: beyond proof of principle.," J. Am. Chem. Soc. 136(6), 2178-2191 (2014).

[18] Broichhagen, J., Frank, J. A., Trauner, D., “A Roadmap to Success in Photopharmacology,” Acc. Chem. Res. 48(7), 1947-1960 (2015).

[19] Bregestovski, P., Maleeva, G., Gorostiza, P., "Light-induced regulation of ligand-gated channel activity.," Br. J. Pharmacol. 172(1), 5870 (2017). 
[20] MarvinSketch 17.28.0 (2017), ChemAxon Ltd. (http://www.chemaxon.com).

[21] Prescription Drug Information, Interactions \& Side Effects. https://www.drugs.com/methotrexate.html (accessed May 24, 2019).

[22] Schoenberger, M., Damijonaitis, A., Zhang, Z., Nagel, D., Trauner, D., "Development of a new photochromic ion channel blocker via azologization of fomocaine.," ACS Chem Neurosci 5(7), 514-518 (2014).

[23] Hynes, J. B., Harmon, S. J., Floyd, G. G., Farrington, M., Hart, L. D., Gale, G. R., Washtien, W. L., Susten, S. S., Freisheim, J. H., "Chemistry and antitumor evaluation of selected classical 2,4-diaminoquinazoline analogues of folic acid.," J. Med. Chem. 28(2), 209-215 (1985).

[24] Gorostiza, P., Soler, C., Matera, C., Camarero, N., Libergoli, M., Gomila Juaneda, A., "Photoisomerizable derivatives of dihydrofolate reductase inhibitors," PCT/EP2018/086233.

[25] Lee, M. S., Bonner, J. R., Bernard, D. J., Sanchez, E. L., Sause, E. T., Prentice, R. R., Burgess, S. M., Brody, L. C., "Disruption of the folate pathway in zebrafish causes developmental defects," BMC Developmental Biology 2012 12:1 12(1), 12, BioMed Central (2012). 